Pacific Journal of Mathematics

“cLsssmicatrov of EeNtrer.oc 


\title{
A CLASSIFICATION OF CENTER-FOCI
}

\author{
ROGER C. McCANN
}

The main purpose of this paper is to classify center-foci according to isomorphisms. Necessary and sufficient conditions are found for an isomorphism to exist in terms of properties on the cycles in suitable neighborhoods of the center-foci. In the last section $o+$ stable isolated critical points are classified according to isomorphisms.

This paper was motivated by discussions with Otomar Hájek and Taro Ura.

Throughout this paper $R^{1}, R^{+}, R^{-}$, and $R^{2}$ will denote the reals, the nonnegative reals, the nonpositive reals, and the plane respectively.

Let $F$ be a family of curves filling a region $R$ of the plane. $F$ will be said to be regular at a point $p$ of $R$ if $F$ is locally homeomorphic with parallel lines at $p . \quad F$ is called regular in $R$ if it is regular at each point of $R$. A cross-section of $F$ (through the point $p$ of $R$ ) shall mean an arc $T$ (of which $p$ is a nonend-point) which lies in $R$ and is such that each curve of $F$ intersects $T$ at most once.

Let $(X, \pi)$ be a dynamical system on $X$, i.e., $X$ is a topological space and $\pi$ is a mapping of $X \times R^{1}$ onto $X$ satisfying the following axioms: (where $x \pi t=\pi(x, t)$ for $(x, t) \in X \times R^{1}$ )

(1) $x \pi 0=x$ for $x \in X$

(2) $(x \pi t) \pi s=x \pi(t+s)$ for $x \in X$ and $t, s \in R^{1}$

(3) $\pi$ is continuous.

For $x \in X, x \pi R^{1}$ is called the trajectory through $x$ and is denoted by $C(x)$. If $C(x)=\{x\}, x$ is called a critical point. If there exists $t \in R^{1}$, $t \neq 0$, such that $x \pi t=x$, then $x$ is called a periodic point. If $x$ is a periodic point and not a critical point, $C(x)$ is called a cycle. A subset $\mathrm{A}$ of $X$ is said to be invariant if $A \pi R^{1}=A$, or + invariant if $A \pi R^{+}=A$. A subset $B$ of $X$ is said to be

(1) orbitally stable (o stable) if $B$ has arbitrarily small invariant neighborhoods.

(2) orbitally + stable $(o+$ stable $)$ if $B$ has arbitrarily small + invariant neighborhoods.

(3) asymptotically orbitally + stable $(a o+$ stable $)$ if $B$ is $o+$ stable and for some neighborhood $U$ of $x, L^{+}(x) \subset \bar{B}$ for every $x \in U$, where $L^{+}(x)$ is the positive limit set of $x$.

Let $(X, \pi)$ be a dynamical system on a metric space $X$. The positive prolongational limit set, denoted by $J^{+}(x)$, of a point $x \in X$ is given by $J^{+}(x)=\left\{y\right.$ : there exist sequences $\left\{x_{i}\right\} \subset X$ and $\left\{t_{i}\right\} \subset R^{1}$ such that $x_{i} \rightarrow x, t_{i} \rightarrow+\infty$, and $\left.x_{i} \pi t_{i} \rightarrow y\right\}$. The negative prolongational limit 
set, $J^{-}(x)$, is defined analogously. For any $x \in X, J^{+}(x)$ and $J^{-}(x)$ are invariant. If $A$ and $B$ are subsets of $X$ such that $A \subset J^{+}(B)\left(A \subset J^{-}(B)\right)$, we will write $A J^{+} B\left(A J^{-} B\right)$.

1. Definition and properties of a center-focus. In the following $\left(R^{2}, \pi\right)$ will denote a dynamical system on $R^{2}$ and $M$ the set of noncritical periodic points of $\left(R^{2}, \pi\right)$.

Definition 1.1. A cycle $C(x)$ of $\left(R^{2}, \pi\right)$ decomposes $R^{2}$ into two components, one bounded and the other unbounded. int $C(x)$ and ext $C(x)$ will denote the bounded and unbounded components, respectively, of $R^{2}-C(x)$.

Proposition 1.2. Let $C(x)$ be a cycle in $\left(R^{2}, \pi\right)$. Then int $C(x)$ and ext $C(x)$ are invariant.

Proof. The components of an invariant set are invariant.

In $[1$, VII, 4.8] it is proved that

Proposition 1.3. If $C(x)$ is a cycle in $\left(R^{2}, \pi\right)$, then int $C(x)$ contains a critical point.

DEFINITION 1.4. An isolated critical point $p$ of $\left(R^{2}, \pi\right)$ is called a center-focus if and only if there exist both cycles and noncyclic trajectories arbitrarily near $p$.

EXAMPLE 1.5. Take any closed set $F$ in $[0,1] \subset R^{+}$and choose any mapping $f: R^{+} \rightarrow[-1,1]$ of class $C^{1}$ having $F$ as its zero-point set. Then define the differential dynamical system by means of the equation.

$$
\frac{d z}{d \theta}=z(i+f|z|) \text {. }
$$

This corresponds to the "polar" equation $(d r / d \theta)=r f(r)$. The origin is an isolated critical point and the cycles of the dynamical system are precisely those circles $|z|=r$ for which $f(r)=0$. We can now make the origin a center-focus by choosing $F$ such that $0 \in F$ and $0 \in \overline{[0,1]-F}$, e.g.,

$$
F=\{0\} \cup\left\{\frac{1}{m}: m \text { is a positive integer }\right\} .
$$

In [1, VIII, 4.1], it is proved that

Proposition 1.6. Let $p$ be a center-focus of $\left(R^{2}, \pi\right)$. Then $p$ is o stable. 
Proposition 1.7. Let $p$ be a center-focus of $\left(R^{2}, \pi\right)$. Then for any $x \in M$ sufficiently near $p, p \in \operatorname{int} C(x)$.

Proof. Let $U$ be a disc neighborhood of $p$ which contains no critical points other than $p$. Since $p$ is $o$ stable, there exists a neighborhood $V$ of $p$ such that $V \pi R^{1} \subset U$. For any $x \in M \cap V, C(x) \subset U$. Then int $C(x) \subset U$ since $U$ is simply connected. By Proposition 1.3, int $C(x)$ contains a critical point. This critical point must be $p$ since $U-\{p\}$ contains no critical points.

COROLLARY 1.8. Let $p$ be a center focus of $\left(R^{2}, \pi\right)$. Then there exists a cycle $C(x)$ such that

(1) $p \in \operatorname{int} C(x)$

(2) $p \in \operatorname{int} C(y)$ for every $y \in \operatorname{int} C(x) \cap M$.

We may now reformulate Definition 1.4 as

DEFINITION 1.4'. An isolated critical point $p$ of $\left(R^{2}, \pi\right)$ is called a center-focus if and only if there exists a cycle $C(x)$ such that

(1) $p$ is the unique critical point in int $C(x)$

(2) there exist both cycles and noncyclic trajectories arbitrarily close to $p$.

We choose a fixed $C\left(x_{0}\right)$ satisfying these conditions and denote int $C\left(x_{0}\right)$ by $U$.

REMARK. The set $\{y: y=p$ or $y \in M \cap \bar{U}\}$ is closed.

The proofs of the following relationships between the cycles in $U$ are the same as the proofs of $[4,1.10]$ and $[4,1.11]$.

Proposition 1.9. Let $p$ be a center-focus and $U$ a neighborhood of $p$ as described in Definition 1.4'. If $C_{1}$ and $C_{2}$ are distinct cycles in $U$, then $C_{1} \subset \operatorname{int} C_{2}$ or $C_{2} \subset \operatorname{int} C_{1}$.

CoRollary 1.10. If $C_{1}$ and $C_{2}$ are distinct cycles in $U$ such that $C_{1} \subset \operatorname{ext} C_{2}$, then $C_{2} \subset \operatorname{int} C_{1}$.

\section{Isomorphism of dynamical systems.}

Definition 2.1. Let $(X, \pi)$ and $(Y, \rho)$ be dynamical systems. A homeomorphism $h: X \rightarrow Y$ is said to be trajectory preserving if and only if $h\left(x \pi R^{1}\right)$ is a trajectory of $(Y, \rho)$ for every $x \in X$. If such a homeomorphism exists, $(X, \pi)$ and $(Y, \rho)$ are said to be equivalent. 
Definition 2.2. Let $(X, \pi)$ and $(Y, \rho)$ be dynamical system. An isomorphism of $(X, \pi)$ onto $(Y, \rho)$ is a pair of mappings $(h, \varphi)$ which satisfies the following conditions:

(1) $h$ is a homeomorphism of $X$ onto $Y$

(2) $\varphi$ is a continuous mapping of $X \times R^{1}$ onto $R^{1}$ such that for every fixed $x \in X, \varphi(x, \cdot)$ is a homeomorphism of $R^{1}$ onto itself with $\varphi(x, 0)=0$

(3) $h(x \pi t)=h(x) \rho \varphi(x, t)$ for $x \in X$ and $t \in R^{1}$.

If such a pair of mappings exists, $(X, \pi)$ and $(Y, \rho)$ are said to be isomorphic.

REMARKs. 1. If $(h, \varphi)$ is an isomorphism of $(X, \pi)$ onto $(Y, \rho)$, then $h$ is a trajectory preserving homeomorphism of $(X, \pi)$ onto $(Y$, $\rho)$. Hence, if $(X, \pi)$ and $(Y, \rho)$ are isomorphic, then they are equivalent.

2. "Equivalent to" is evidently an equivalence relation in the family of all dynamical systems.

3. In [5], it is proved that "isomorphic to" is an equivalence relation in the family of all dynamical systems.

The following theorem, [6, 3, Th. 3], states conditions for "equivalent to" and "isomorphic to" to be identical.

THEOREM 2.3. Let $(X, \pi)$ and $(Y, \rho)$ be dynamical systems on completely regular Hausdorff spaces $X$ and $Y$ and let $S_{\pi}$ and $S_{\rho}$ denote the set of critical points of $(X, \pi)$ and $(X, p)$ respectively. Then if $h$ is a trajectory preserving homeomorphism of $(X, \pi)$ onto $(Y, \rho)$ there exists a mapping $\varphi:\left(X-S_{\pi}\right) \times R^{1} \rightarrow R^{1}$ such that $\left(h \mid X-S_{\pi}, \varphi\right)$ is an isomorphism of $\left(X-S_{\pi}, \pi\right)$ onto $\left(Y-S_{\rho}, \rho\right)$.

Let $(h, \varphi)$ be an isomorphism of $(X, \pi)$ onto $(Y, \rho)$. If $X$ is connected, it can be shown that $\varphi(x, \cdot)$ is either strictly increasing for every $x \in X$ or strictly decreasing for every $x \in X$.

Definition 2.4 Let $(X, \pi)$ and $(Y, \rho)$ be dynamical systems on connected spaces $X$ and $Y$ and let $(h, \varphi)$ be an isomorphism of $(X, \pi)$ onto $(X, \rho)$. If $\varphi(x, \cdot)$ is strictly increasing for every $x \in X,(h, \varphi)$ is called proper. If $\varphi(x, \cdot)$ strictly decreasing for every $x \in X,(h, \varphi)$ is called dual.

The following proposition displays the connection between isomorphisms and prolongational limit sets.

Proposition 2.5. Let $(X, \pi)$ and $(Y, \rho)$ be dynamical systems on connected metric spaces $X$ and $Y$ and let $(h, \varphi)$ be an isomorphism of $(X, \pi)$ onto $(Y, \rho)$. For $x \in X$ 
(1) if $(h, \varphi)$ is proper, then $h\left(J_{\pi}^{+}(x)\right)=J_{\rho}^{+}(h(x))$

(2) if $(h, \varphi)$ is dual, then $h\left(J_{\pi}^{+}(x)\right)=J_{\rho}^{-}(h(x))$, where the subscript $\pi$ or $\rho$ denotes the dynamical system with respect to which the positive or negative prolongational limit set was taken.

Proof. The proof follows from the definitions of proper and dual.

3. Curve families of Kaplan. In [3] Kaplan classifies families in a neighborhood of a singularity. In particular, if we consider the trajectories of a planar dynamical system as curves, he classifies the dynamical system in a neighborhood of an isolated critical point. For a singularity surrounded by closed curves, the classification is done as follows.

Definition 3.1. Let $A$ and $B$ be curve families filling the planar sets $U$ and $V$ respectively. $A$ and $B$ are said to be equivalent if there exists an orientation preserving homeomorphism $h: U \rightarrow V$ such that for any curve $C$ of $A, h(C)$ is a curve of $B$.

DeFinition 3.2. An annular family shall mean a curve family $F$ in the plane which has the following properties:

(1) $F$ fills a closed region $A$, whose boundary consists of two disjoint closed curves $D_{1}$, and $D_{2}$.

(2) $F$ is regular at every point of $A$.

A half open annular family shall mean a family which can be written as a union $\bigcup_{i=1}^{\infty} F_{i}$ of closed annular families $F_{i}$, where $F_{i} \cap F_{i+1}$ is the common bounding curve of $F_{i}$ and $F_{i+1}$ for each $i$ and $F_{i} \cap F_{j}$ is the empty set for $|i-j|>1$.

REMARK. If $F$ is a curve family defined by a planar dynamical system without critical points, then $F$ is regular. This is an immediate consequence of the existence of a transversal through each point of the dynamical system. [1, VI, 2.12; VIII, 1.6]

Definition 3.3. An annular family $F$ will be called simple if it contains no closed curves besides $D_{1}$ and $D_{2}$.

The possibilities for a simple annular family are of two fundamental types: the type of spirals which are asymptotic to the boundary curves in the opposite direction; the type of spirals which are asymptotic to the boundary curves in the same direction.

Kaplan shows that a simple annular family may be mapped by an orientation preserving homeomorphism onto one of the following four annular families.

(i) the family of Figure 1. 
(ii) the family obtained from (i) by reflecting in a diameter of the larger circle.

(iii) the family of Figure 2.

(iv) the family obtained from (iii) by reflecting in a diameter of the larger circle.

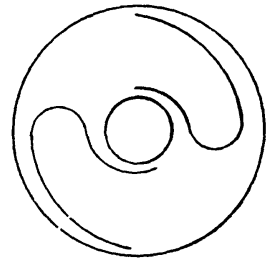

Figure 1.

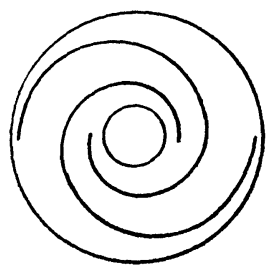

Figure 2.

Families which are homeomorphic to (i) or (ii) are called bent. An annular family can contain only a finite number of bent families. Families which are homeomorphic to (iii) or (iv) are called smooth. A smooth annular family is an annular family which contains no bent families.

Every half open annular family can be associated with a sequence of letters such as:

$$
b s b b b s b \cdots \text { or } \quad s b s b s b \ldots
$$

corresponding to successive rings of bent or smooth types. The sequence is made unique by writing the letters corresponding to the rings in order of nearness of the ring to the singular point.

With each of the $b$ 's is associated a bent annular family. This bent annular family has an associated canonical family (i) or (ii). We now insert after each $b$ in the sequence the corresponding symbol $i$ or $i i$ as superscripts, so that the symbol might appear

$$
b^{i} s b^{i} b^{i i} s b^{i i} s . .
$$

DEFINITION 3.4. Let $h_{1}$ and $h_{2}$ be two orientation preserving homeomorphisms of $0 \leqq y \leqq 1$ onto itself. $h_{1}$ and $h_{2}$ are called similar if and only if the following conditions holds:

(1) If $E_{i}$ is the (necessarily closed) set of fixed points of $h_{i}(i=$ $1,2)$, then there exists a homeomorphism $f: E_{1} \rightarrow E_{2}$ which is a monotone increasing function.

(2) If $y_{i}^{\prime}$ and $y_{i}^{\prime \prime}$ being to $E_{i}$, but $y \notin E_{i}$ for $y_{i}^{\prime} \leqq y \leqq y_{i}^{\prime \prime}$ and $f\left(y_{1}^{\prime}\right)=y_{2}^{\prime}, f\left(y_{1}^{\prime \prime}\right)=y_{2}^{\prime \prime}$, then $h_{1}(y)-y$ and $h_{2}(y)-y$ have the same sign in the respective intervals $y_{i}^{\prime} \leqq y \leqq y_{i}^{\prime \prime}$.

To each smooth annular family there exists a cross-section, [3, Corollary 1, p. 27]. If a smooth annular family (bounded by $D_{1}$ and $D_{2}$ ) 
is cut open along a cross section and mapped onto a rectangular family $M=\{0 \leqq x \leqq 1,0 \leqq y \leqq 1$, the "curves" being the lines $y=a$ constant\} so that $D_{1}$ corresponds to $y=0$ and $D_{2}$ to $y=1$ and the direction from $(0,0)$ to $(1,0)$ on $y=0$ is taken to be the positive orientation on $D_{1}$, then the corresponding identification is determined up to a class of similar homeomorphisms.

Suppose the set of all orientation preserving homeomorphisms of $0 \leqq y \leqq 1$ onto itself is subdivided into similarily classes. To each class assign a different index $\alpha$, so that a collection $I$ of indices is formed in one-to-one correspondence with the similarily classes. Then to each smooth annular family there corresponds a unique index of $I$. Moreover, two smooth annular families have the same index if and only if they are equivalent. We now insert into our already modified symbol for the smooth annular family the corresponding index of $I$ after each $s$, so that the symbol might appear

$$
b^{i} s(\alpha) b^{i i} b^{i} s(\beta) \cdots .
$$

Let $F$ be an annular family. Then $b s(F)$ will denote the symbol corresponding to $F$. The theorem of Kaplan in which we are interested is ([3, Th. 22a])

Theorem 3.5. Let $F$ and $G$ be two half-open annular families. Then $F$ and $G$ are equivalent if and only if $b s(F)=b s(G)$.

4. Classification of center-foci. Kaplan looked at the behavior of all the curves to obtain his classification. We will now apply his theorem to show that it is necessary and sufficient to consider relations between the cycles of two center-foci in order to obtain a classification.

For $i=1,2$, let $\left(R^{2}, \pi_{i}\right)$ be a dynamical system, $p_{i}$ a center-focus and $U_{i}$ a neighborhood of $p_{i}$ as described in Definition 1.4'. By restricting the mapping $\pi_{i}$ to $U_{i}$ we may obtain a dynamical system $\left(U_{i}, \pi_{i} \mid U_{i}\right)$ which will be denoted by $\left(U_{i}, \pi_{i}\right)$. For $x \in R^{2}, C_{i}(x)$ and $J_{i}^{+}\left(J_{i}^{-}\right)$ will denote the trajectory and the positive (negative) prolongational limit set with respect to $\left(R^{2}, \pi_{i}\right), i=1,2 . \quad M_{i}$ will denote the family of cycles of $\left(U_{i}, \pi_{i}\right), i=1,2$.

LEMMA 4.1. Let $(V, \pi)$ be a dynamical system whose curves form a simple annular family with $D_{1}$ and $D_{2}$ as boundings cycles. Then $D_{1} J^{+} D_{2}$ or $D_{2} J^{+} D_{1}$.

Proof. Let $x$ be any point in the interior of $V$. Then $C(x)$ is asymptotic to both $D_{1}$ and $D_{2}$. Let $x \pi R^{+}$be asymptotic to $D_{1}$ and $x \pi R^{-}$be asymptotic to $D_{2}$. If the reverse is true, the proof is similar. 
For $i=1,2$, if $z_{i}$ is any point of $D_{i}$, then there exists a sequence $t_{m}^{i} \in R^{1}$ such that $x \pi t_{m}^{i} \rightarrow z_{i}$. Moreover $t_{m}^{1} \rightarrow+\infty$ and $t_{m}^{2} \rightarrow-\infty$. Then $t_{m}^{1}-t_{m}^{2} \rightarrow+\infty, x \pi t_{m}^{2} \rightarrow z_{2}$, and

$$
\left(x \pi t_{m}^{2}\right) \pi\left(t_{m}^{1}-t_{m}^{2}\right)=x \pi t_{m}^{1} \rightarrow z_{1} .
$$

Thus $z_{1} \in J^{+}\left(z_{2}\right)$ and $D_{1} J^{+} D_{2}$.

Definition 4.2. A homeomorphism $f: M_{1} \rightarrow M_{2}$ is said to preserve the order of cycles if and only if $f$ satisfies the following property for $C_{1}, C_{2} \in M_{1}$ :

$$
C_{1} \subset \operatorname{int} C_{2} \text { if and only if } f\left(C_{1}\right) \subset \operatorname{int} f\left(C_{2}\right) \text {. }
$$

Definition 4.3. The orientation of the cycles of $M_{i}, i=1,2$, will be in the direction of movement with respect to $R^{+}$.

Proposition 4.4. $\left(U_{1}-p_{1}, \pi_{1}\right)$ and $\left(U_{2}-p_{2}, \pi_{2}\right)$ are isomorphic if there exists a homeomorphism $f: M_{1} \rightarrow M_{1}$ such that

(1) $f$ preserves the order of cycles

(2) $f$ is orientation preserving

(3) $C_{1} J_{1}^{+} C_{2}$ if and only if $f\left(C_{1}\right) J_{2}^{+} f\left(C_{2}\right)$ for $C_{1}, C_{2} \in M_{1}$.

Proof. We will first show that to each simple annular family of $U_{1}$ there corresponds an equivalent simple annular family of $U_{2}$.

Let $F$ be a simple annular family of $U_{1}$ with $D_{1}$ and $D_{2}, D_{1} \subset \operatorname{int} D_{2}$, as the cycles which bound $F$. For definiteness let $F$ be equivalent to (i) and $D_{2} J_{1}^{+} D_{1}$. If $F$ is equivalent to one of the other canonical families or if $D_{1} J_{1}^{+} D_{2}$, the proof is similar.

$D_{1}$ and $D_{2}$ are oriented in opposite directions since the spirals of $F$ are asymptotic to $D_{1}$ and $D_{2}$ in opposite directions. Since $F$ is equivalent to (i), we will, for simplicity, assume $F$ is (i). Then the spirals of $F$ are asymptotic to $D_{1}$, in a counter-clockwise direction, i.e., if we move along one of the spirals in a counter-clockwise direction we can come arbitrarily close to $D_{1}$. Since $D_{2} J_{1}^{+} D_{1}$, the orientation of $D_{1}$ must be in a clockwise direction. The orientation of $D_{2}$ must then be in a counter-clockwise direction.

Let $G$ be the family of curves bounded by $f\left(D_{1}\right)$ and $f\left(D_{2}\right)$. By (1), $G$ can contain no cycles other than $f\left(D_{1}\right)$ and $f\left(D_{2}\right)$. $\quad G$ is a simple annular family. By (2), $f\left(D_{1}\right)$ and $f\left(D_{2}\right)$ are oriented in the same directions as $D_{1}$ and $D_{2}$ respectively. Thus $f\left(D_{1}\right)$ and $f\left(D_{2}\right)$ are oriented in a clockwise and counter-clockwise direction respectively. Then the spirals of $G$ are asymptotic to $D_{1}$ and $D_{2}$ in opposite directions, i.e., $G$ is a bent region. If the spirals of $G$ are asymptotic to $f\left(D_{1}\right)$ and $f\left(D_{2}\right)$ in the same directions that $f\left(D_{1}\right)$ and $f\left(D_{2}\right)$ are oriented, $G$ is 
equivalent to family represented by Figure 4 . If the spirals of $G$ are asymptotic to $f\left(D_{1}\right)$ and $f\left(D_{2}\right)$ in the opposite directions that $f\left(D_{1}\right)$ and $f\left(D_{2}\right)$ are oriented, $G$ is equivalent to the family represented in Figure 3. Figure 3 represents (i) and Figure 4 represents (ii).

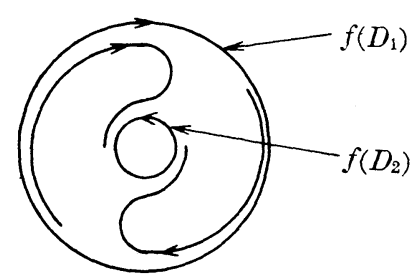

Figure 3.

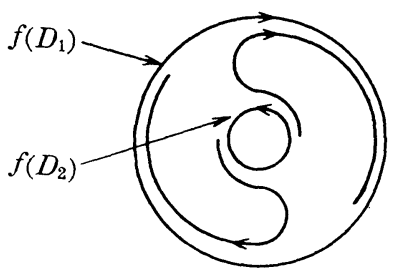

Figure 4 .

$f\left(D_{1}\right) \subset \operatorname{int} f\left(D_{2}\right)$ and $f\left(D_{1}\right) J_{2}^{+} f\left(D_{2}\right)$ by (1) and (3) respectively. Since $f\left(D_{1}\right) J_{2}^{+} f\left(D_{2}\right)$, the spirals must be asymptotic to $f\left(D_{1}\right)$ and $f\left(D_{2}\right)$ in the opposite directions that $f\left(D_{1}\right)$ and $f\left(D_{2}\right)$ are oriented. Hence $G$ is equivalent to (i) and therefore equivalent to $F$.

We will now show that to each smooth annular family in $U$, there corresponds an annular family in $U_{2}$ with the same index. Let $F$ be a smooth annular family in $U_{1}$ with bounding cycles $D_{3}$ and $D_{4}$, $D_{3} \subset \operatorname{int} D_{4}$. All the cycles in $F$ have the same orientation. Otherwise $F$ would contain a bent annular family. Let $G$ be the annular family bounded by $f\left(D_{3}\right)$ and $f\left(D_{4}\right) . \quad G$ is smooth since all cycles are oriented in the same direction. Let $S_{1}$ and $S_{2}$ be cross-sections of $F$ and $G$ respectively. We may define a mapping $T_{i}: S_{i} \rightarrow S_{i}$ as follows:

$$
T_{i}(x)=\left\{\begin{array}{l}
x \quad \text { if } \quad C_{i}(x) \in M_{i} \\
x \pi_{i} t(x) \text { if } \quad C_{i}(x) \in M_{i}
\end{array}\right.
$$

where $t(x) \in R^{+}$is such that $x \pi_{i} t(x) \in S_{i}$ and $x \pi_{i}(0, t(x)) \cap S_{i}=\phi$. Evidently $T_{i}$ is one-to-one and onto. The continuity of $T_{i}$ follows from the fact that for a given $x \in S_{i}$, subares of $S_{i}$ containing $x$ as a nonend-point generate arbitrarily small neighborhoods of $x$, i.e., if $A$ is any subarc of $S_{i}$ containing $x$ as a nonend-point, $A \pi_{i}(-a, a)$ is a neighborhood of $x$ for $a>0$. Hence $T_{i}$ is a homeomorphism of $S_{i}$ onto itself whose fixed points correspond to the intersection of the cycles of $\left(U_{i}, \pi_{i}\right)$ with $S_{i}, i=1,2$.

For $i=1,2$, let $f_{i}$ be a homeomorphism of $S_{i}$ onto $[0,1]$ such that $f_{1}\left(D_{3} \cap S_{1}\right)=0$ and $f_{2}\left(f\left(D_{3}\right) \cap S_{2}\right)=0$. Let

$$
x_{1}, x_{2} \in M_{1} \cap S_{1}, C_{1}\left(x_{2}\right) \subset \operatorname{int} C_{1}\left(x_{1}\right),
$$

and $y \notin M_{1} \cap S_{1}$ for $y$ in the subarc of $S_{1}$ with end-points $x_{1}$ and $x_{2}$. Then $C_{1}\left(x_{1}\right) \cup C_{1}\left(x_{2}\right)$ forms the boundary of a simple annular family. Therefore $C_{1}\left(x_{1}\right) J_{1}^{+} C_{1}\left(x_{2}\right)$ or $C_{1}\left(x_{2}\right) J_{1}^{+} C_{1}\left(x_{1}\right)$. For definiteness let 
$C_{1}\left(x_{1}\right) J_{1}^{+} C_{1}\left(x_{2}\right)$. The proof is similar if $C_{1}\left(x_{2}\right) J_{1}^{+} C_{1}\left(x_{1}\right)$. Then $y \pi_{i} t_{i}(y)$ $\left(=T_{1}(y)\right)$ intersects $S_{1}$ between $y$ and $x_{1}$ by the Monotonicity Theorem [1, VIII, 4.13]. Then $f_{1} T_{1}(y)-f_{1}(y)$ has constant sign for $y$ in the subarc of $S_{1}$ with end-points $x_{1}$ and $x_{2}$. Similarly $f_{2} T_{2}(y)-f_{2}(y)$ has the same sign for all $y$ in the subare of $S_{2}$ with end-points $f\left(C_{1}\left(x_{1}\right)\right) \cap S_{2}$ and $f\left(C_{2}\left(x_{2}\right)\right) \cap S_{2}$. Since $D_{3} \subset \operatorname{int} D_{4}, f_{1}\left(D_{3} \cap S_{1}\right)=0$, and $f_{1}\left(D_{4} \cap S_{1}\right)=1$, $f$ increases from $x_{2}$ to $x_{1}$ along $S_{1}$. Similarily $f_{2}$ increases from $f\left(C_{2}\left(x_{1}\right)\right) \cap S_{2}$ to $f\left(C_{1}\left(x_{2}\right)\right) \cap S_{2}$ along $S_{2}$. Then $f_{1} T_{1}(y)-f_{1}(y)$ and $f_{2} T_{2}(y)-f_{2}(y)$ are both positive for $y$ in the appropriate subarc. Hence $T_{1}$ and $T_{2}$ are similar. $F$ and $G$ are equivalent.

We have shown that to each bent or smooth annular family of $U_{1}$ there corresponds an equivalent bent or smooth annular family of $U_{2}$. The ordering (with respect to nearness to the critical point) of the annular families of $U_{1}$ is the same as the ordering of the equivalent annular families of $U_{2}$ because the order of the boundaries of the annular families is preserved by (1). Hence $b s\left(U_{1}\right)=b s\left(U_{2}\right)$. By Theorem 3.5, $\left(U_{1}, \pi_{1}\right)$ and $\left(U_{2}, \pi_{2}\right)$ are equivalent. Theorem 2.3 yields the desired result.

REmaRks. 1. If we replace (2) and (3) by $\left(2^{\prime}\right)$ and $\left(3^{\prime}\right)$ the conclusion of Proposition 4.4 remains valid.

(2') $f$ is orientation reversing

(3') $C_{1}, J_{1}^{+} C_{2}$ if and only if $f\left(C_{1}\right) J_{2}^{-} f\left(C_{2}\right)$ for $C_{1}, C_{2} \in M_{1}$. The proof is analogous to that of Proposition 4.3.

2. Conditions (2) and (3) are independent as the following examples show. Let $F$ and $G$ be the simple annular families indicated by Figures 5 and 6.

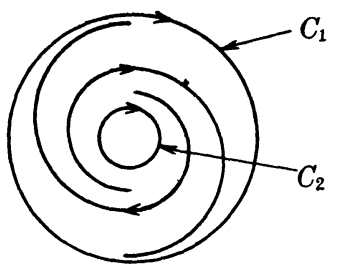

Figure 5 .

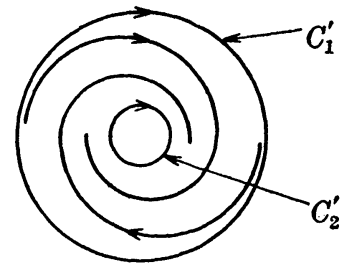

Figure 7.

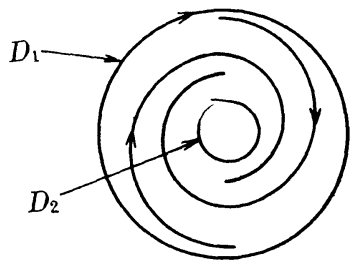

Figure 6.

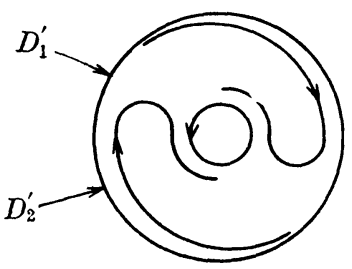

Figure 8. 
Let $f\left(C_{i}\right)=D_{i}$. Then (2) is satisfied and (3) is not.

Now let $F^{\prime}$ and $G^{\prime}$ be the simple annular families indicated by Figures 7 and 8.

Let $f\left(C_{i}^{\prime}\right)=D_{i}^{\prime}$. Then (3) is satisfied and (2) is not.

THEOREM 4.5. $\left(U_{1}-\left\{p_{1}\right\}, \pi_{1}\right)$ and $\left(U_{2}-\left\{p_{2}\right\}, \pi_{2}\right)$ are isomorphic if and only if either (1), (2) and (3) or (1), $\left(2^{\prime}\right)$, and $\left(3^{\prime}\right)$ are satisfied.

Proof. If: This is Proposition 4.4 and Remark 1 following Proposition 4.4.

Only If: Let $(h, \varphi)$ be an isomorphism of $\left(U_{1}-\left\{p_{1}\right\}, \pi_{1}\right)$ onto $\left(U_{2}-\left\{p_{2}\right\}, \pi_{2}\right)$. Then $h$ defines a homeomorphism $h^{\prime}: M_{1} \rightarrow M_{2}$. $h$ may be extended to a homeomorphism of $U_{1}$ onto $U_{2}$ by defining $h\left(p_{1}\right)=p_{2}$. Hence we may assume $h$ is a homeomorphism of $U_{1}$ onto $U_{2}$. Let $C_{1}$, $C_{2} \in M_{1}, C_{1} \subset \operatorname{int} C_{2}$. Then $\partial h\left(\operatorname{int} C_{2}\right)=h\left(C_{2}\right)$. This and Corollary 1.10 imply $h\left(C_{1}\right) \subset \operatorname{int} h\left(C_{2}\right)$. Since $h^{\prime}\left(C_{i}\right)=h\left(C_{i}\right), i=1,2, h^{\prime}$ satisfies (1).

We now consider the case that $(h, \varphi)$ is proper. Then $\varphi(x, \cdot)$ is an increasing function implies the orientation of $C$ and $h^{\prime}(C)$ are the same for $C \in M_{1}$. $\quad h^{\prime}$ satisfies (2). By applying Proposition 2.5, $h^{\prime}$ satisfies (3).

If $(h, \varphi)$ is dual, then $\varphi(x, \cdot)$ is a decreasing function and the orientation of $C$ and $h^{\prime}(C)$ are reversed for any $C \in M_{1}$. Proposition 2.5 implies $h^{\prime}$ satisfies (3).

Thus if $(h, \varphi)$ is proper, $(1),(2)$, and (3) are satisfied and if $(h, \varphi)$ is dual, (1), $\left(2^{\prime}\right)$, and $\left(3^{\prime}\right)$ are satisfied.

5. Classification of $o+$ stable isolated critical points. The behavior of trajectories in a sufficiently small neighborhood of an $o+$ stable isolated critical point has been completely determined, [1, VII, 4.1]. The theorem states

THEOREM 5.1. Let $p$ be an o + stable isolated critical point of a dynamical system $(X, \pi)$. Then $p$ satisfies precisely one of the following conditions:

(1) $p$ is asymptotically orbitally + stable (a focus).

(2) Some neighborhood of $p$ consists entirely of cycles (a center).

(3) $p$ is a center-focus.

Furthermore $p$ is an $o+$ stable if and only if (1) holds, and o stable if and only if (2) or (3) hold.

We have just classified center-foci. Centers are classified in [4]. To complete the classification of an $o+$ stable critical point, we must classify foci.

In [1, VII, 4.3] it is proved that 
Proposition 5.1. Each o + stable isolated critical point, not a center, possesses arbitrarily small neighborhoods bounded by closed transversal curves.

For $i=1,2$, let $p_{i}$ be a focus of $\left(R^{2}, \pi_{i}\right), A_{i}$ be the region of attraction $([1, I V, 4.9])$ for $p_{i}$, and $S_{i}$ be a closed transversal curve contained in $A_{i}$. Then it can be shown that $S_{i} \pi_{i} R^{1}=A_{i}-\left\{p_{i}\right\}, i=1,2$, i.e., $A_{i}-\left\{p_{i}\right\}$ is parallelizable.

Proposition 5.2. $\left(A_{1}, \pi_{1}\right)$ and $\left(A_{2}, \pi_{2}\right)$ are isomorphic.

Proof. Let $f: S_{1} \rightarrow S_{2}$ be a homeomorphism. Then for each $x \in A_{1}-\left\{p_{1}\right\}$ there exists a unique $t(x)$ such that $x \pi_{1} t(x) \cap S_{1} \quad(1$, VII, 4.4]). Moreover since $A_{i}-\left\{p_{i}\right\}$ is parallelizable, $t(\cdot)$ is a continuous mapping of $A_{1}-p_{1}$ onto $R^{1}$.

It can be verified that

$$
h(x)=\left\{\begin{array}{lll}
f\left(x \pi_{1} t(x)\right) \pi_{2}-t(x) & \text { if } \quad x \neq p_{1} \\
p_{2} & \text { if } \quad x=p_{1}
\end{array}\right.
$$

is a homeomorphism. Noting that $t\left(x \pi_{1} s\right)=t(x)-s$ we get that $h\left(x \pi_{1} s\right)=h(x) \pi_{2} s$ for $x \in A_{1}$ and $s \in R^{1}$. If we denote the identity mapping of $R^{1}$ onto itself by $i,(h, i)$ is an isomorphism of $\left(A_{1}, \pi_{1}\right)$ onto $\left(A_{2}, \pi_{2}\right)$.

\section{REFERENCES}

1. Otomar Hájek, Dynamical systems in the plane, Academic Press, London, 1968.

2. - Categorical concepts in dynamical systems theory, Proceedings of the Topological Dynamics Symposium, Fort Collins, Colorado, W. A. Benjamin, 1968.

3. Wilfred Kaplan, The structure of a curve-family on a surface in the neighborhood of an isolated singularity, Amer. J. Math. 64 (1942), 1-35.

4. Roger C. McCann, A classification of centers, Pacific J. Math. (to appear)

5. Taro Ura, Local isomorphisms and local parallelizability in dynamical systems theory, Mathematics Systems Theory 2 (1968).

6. - Isomorphism and local characterization of local dynamical systems (to appear)

Received April 7, 1969.

California State College at los angeles 


\section{PACIFIC JOURNAL OF MATHEMATICS}

\section{EDITORS}

\author{
H. SAMELSON \\ Stanford University \\ Stanford, California 94305 \\ RICHARD PIERCE \\ University of Washington \\ Seattle, Washington 98105
}

J. DugundJI

Department of Mathematics University of Southern California Los Angeles, California 90007

BASIL GORDON*

University of California

Los Angeles, California 90024

\section{ASSOCIATE EDITORS}
E. F. BECKENBACH
B. H. NEUMANN
F. WOLE
K. YOSHIDA

\section{SUPPORTING INSTITUTIONS}

\author{
UNIVERSITY OF BRITISH COLUMBIA \\ CALIFORNIA INSTITUTE OF TECHNOLOGY \\ UNIVERSITY OF CALIFORNIA \\ MONTANA STATE UNIVERSITY \\ UNIVERSITY OF NEVADA \\ NEW MEXICO STATE UNIVERSITY \\ OREGON STATE UNIVERSITY \\ UNIVERSITY OF OREGON \\ OSAKA UNIVERSITY \\ UNIVERSITY OF SOUTHERN CALIFORNIA
}

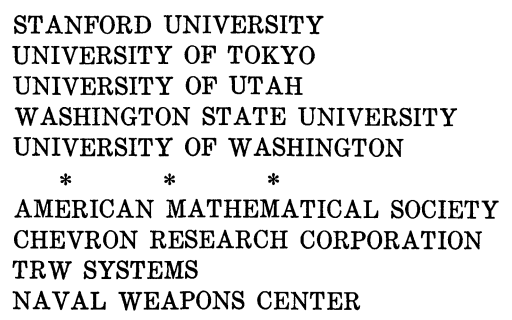

The Supporting Institutions listed above contribute to the cost of publication of this Journal, but they are not owners or publishers and have no responsibility for its content or policies.

Mathematical papers intended for publication in the Pacific Journal of Mathematics should be in typed form or offset-reproduced, (not dittoed), double spaced with large margins. Underline Greek letters in red, German in green, and script in blue. The first paragraph or two must be capable of being used separately as a synopsis of the entire paper. The editorial "we" must not be used in the synopsis, and items of the bibliography should not be cited there unless absolutely necessary, in which case they must be identified by author and Journal, rather than by item number. Manuscripts, in duplicate if possible, may be sent to any one of the four editors. Please classify according to the scheme of Math. Rev. 36, 1539-1546. All other communications to the editors should be addressed to the managing editor, Richard Arens, University of California, Los Angeles, California, 90024.

50 reprints are provided free for each article; additional copies may be obtained at cost in multiples of 50 .

The Pacific Journal of Mathematics is published monthly. Effective with Volume 16 the price per volume (3 numbers) is $\$ 8.00$; single issues, $\$ 3.00$. Special price for current issues to individual faculty members of supporting institutions and to individual members of the American Mathematical Society: $\$ 4.00$ per volume; single issues $\$ 1.50$. Back numbers are available.

Subscriptions, orders for back numbers, and changes of address should be sent to Pacific Journal of Mathematics, 103 Highland Boulevard, Berkeley, California, 94708.

PUBLISHED BY PACIFIC JOURNAL OF MATHEMATICS, A NON-PROFIT CORPORATION

Printed at Kokusai Bunken Insatsusha (International Academic Printing Co., Ltd.), 7-17, Fujimi 2-chome, Chiyoda-ku, Tokyo, Japan.

* Acting Managing Editor. 


\section{Pacific Journal of Mathematics}

\section{Vol. 32, No. $2 \quad$ February, 1970}

Harry P. Allen and Joseph Cooley Ferrar, Jordan algebras and exceptional subalgebras of the exceptional algebra $E_{6} \ldots \ldots \ldots \ldots \ldots \ldots \ldots 283$

David Wilmot Barnette and Branko Grünbaum, Preassigning the shape of a

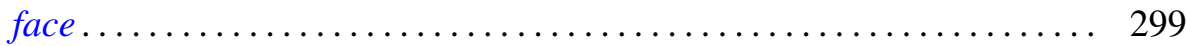

Robert Francis Craggs, Involutions of the 3-sphere which fix 2-spheres . . . . 307

David William Dean, Bor-Luh Lin and Ivan Singer, On k-shrinking and $k$-boundedly complete bases in Banach spaces ................ 323

Martin Engert, Finite dimensional translation invariant subspaces ....... 333

Kenneth Lewis Fields, On the global dimension of residue rings ......... 345

Howard Gorman, The Brandt condition and invertibility of modules ....... 351

Benjamin Rigler Halpern, A characterization of the circle and interval ..... 373

Albert Emerson Hurd, A uniqueness theorem for second order quasilinear

hyperbolic equations ............................... 415

James Frederick Hurley, Composition series in Chevalley algebras ...... 429

Meira Lavie, Disconjugacy of linear differential equations in the complex

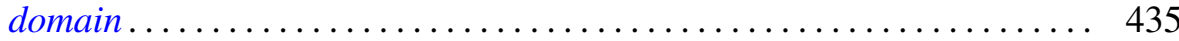

Jimmie Don Lawson, Lattices with no interval homomorphisms ......... 459

Roger McCann, A classification of center-foci ................. 467

Evelyn Rupard McMillan, On continuity conditions for functions . . . . . . . 479

Graciano de Oliveira, A conjecture and some problems on permanents .... 495

David L. Parrott and S. K. Wong, On the Higman-Sims simple group of order $44,352,000$.

Jerome L. Paul, Extending homeomorphisms ................. 517

Thomas Benny Rushing, Unknotting unions of cells .............. 521

Peter Russell, Forms of the affine line and its additive group.......... 527

Niel Shilkret, Non-Archimedean Gelfand theory ................. 541

Alfred Esperanza Tong, Diagonal submatrices of matrix maps.......... 551 